\title{
Inhibition of Nitric Oxide Synthase Does Not Impair Spatial Learning
}

\author{
D. M. Bannerman, ${ }^{1}$ P. F. Chapman, ${ }^{3}$ P. A. T. Kelly, ${ }^{2}$ S. P. Butcher, ${ }^{1}$ and R. G. M. Morris ${ }^{1}$ \\ Departments of ${ }^{1}$ Pharmacology and ${ }^{2}$ Clinical Neuroscience, Centre for Neuroscience, University of Edinburgh Medical \\ School, Edinburgh EH8 9JZ, Scotland, and ${ }^{3}$ Department of Psychology and Graduate Program in Neuroscience, \\ University of Minnesota, Minneapolis, Minnesota 55455
}

Nitric oxide (NO), a putative intercellular messenger in the CNS, may be involved in certain forms of synaptic plasticity and learning. This article reports a series of experiments investigating the effects of $N_{\omega}$-nitro-L-arginine methyl ester (L-NAME) upon various forms of learning and memory in the watermaze. L-NAME (75 $\mathrm{mg} / \mathrm{kg}$, i.p., sufficient to bring about $>\mathbf{9 0} \%$ inhibition of NO synthesis in brain) produced an apparent impairment in spatial learning when given to naive rats during acquisition ( $3 \mathrm{~d}$, six training trials per day). This impairment was dose related, stereoselective, and attenuated by coadministration of L-arginine. A second study showed that L-NAME did not affect the retention of a previously learned spatial task. In addition, in a visual discrimination task, the rate at which criterion levels of performance were reached was unaffected by L-NAME. Thus, inhibition of NO synthase may cause a selective impairment of spatial learning without effect upon retention. However, analysis of the early training trials of the visual discrimination task revealed significantly elevated escape latencies in the L-NAME-treated rats, suggesting that inhibition of NO synthase may have more general effects. As normal rats learn the spatial task very rapidly, the possibility arises that the apparent deficit in learning is due to a disruption of some process other than learning per se. A further series of experiments investigated this possibility. L-NAME was found not to impair the learning of a new platform position in the same spatial environment. Surprisingly, L-NAME also had no effect on spatial learning in a second watermaze located in a novel spatial environment by rats well practiced with all aspects of watermaze training. Finally, L-NAME had no effect on spatial learning in naive rats trained with just one trial per day. Thus, systemic injection of an NO synthase inhibitor impairs behavioral performance in two tasks during their initial acquisition, but the basis of this functional disruption is unlikely to be due to any direct effect upon the mechanisms of spatial learning.

[Key words: nitric oxide, long-term potentiation, hippocampus, rat, watermaze, spatial learning]

Associative long-term potentiation (LTP) is a form of activitydependent synaptic plasticity that is widely believed to participate in learning and memory (Bliss and Lomo, 1973; Morris

\footnotetext{
Received Oct. 13, 1993; revised Apr. 15, 1994; accepted Apr. 27, 1994.

This work was supported by an MRC programme grant, a grant from the Human Frontiers Science Panel (R.G.M.M., D.M.B.), the Whitehall Foundation, a McKnight-Land grant professorship (P.F.C.), and a Wellcome Trust project grant

(P.A.T.K.). We thank Roger Spooner for computing software

Correspondence should be addressed to $\mathrm{D}$. M. Bannerman at the above address. Copyright (C) 1994 Society for Neuroscience $0270-6474 / 94 / 147404-11 \$ 05.00 / 0$
}

et al., 1990; Bliss and Collingridge, 1993). Evidence supporting a role for the mcchanisms of LTP in learning is based, in part, on comparable effects of drugs on LTP and on learning. For example, the induction of LTP requires the activation of NMDA receptors (Collingridge et al., 1983) and it has been shown that the NMDA antagonist D-AP5 impairs spatial learning across a dose range comparable to the inhibition of hippocampal LTP in vivo (Davis et al., 1992). Along similar lines, several studies have shown that inhibitors of NO synthase impair certain forms of learning (Chapman et al., 1992; Hölscher and Rose, 1992; Böhme et al., 1993), and block the induction of LTP in the in vitro hippocampal slice (Böhme et al., 1991; O'Dell et al., 1991; Schuman and Madison, 1991; Haley et al., 1992).

Although our own observations, made in a parallel study (see following companion article, Bannerman et al., 1994), call into question whether L- nitro-arginine methyl ester (L-NAME) blocks the induction of hippocampal LTP in vivo, the possibility that inhibition of NO synthase may limit its induction suggests a novel means to investigate the relationship between LTP and learning. To date, there have been several reports indicating that blocking NO synthase impairs performance in a variety of both spatial and nonspatial learning tasks. Chapman et al. (1992) have shown that intraperitoneal injections of L-NAME $(75 \mathrm{mg} /$ $\mathrm{kg}$ ) impair acquisition of spatial reference memory in the watermaze but do not affect the retention of previously acquired spatial information. Inhibitors of NO synthase have also been reported to impair performance in a spatial reference memory task in the eight-arm radial maze (Böhme et al., 1993). However, the effects of $\mathrm{NO}$ synthase inhibitors on learning do not appear to be restricted to hippocampal-dependent tasks such as spatial learning. Deficits in the acquisition of conditioned eyeblink responses (Chapman et al., 1992) and in one trial inhibitory avoidance in the chick (Hölscher and Rose, 1992) following administration of NO synthase inhibitors have also been reported.

A specific issue to address in investigating the action of NO concerns the specificity of the behavioral impairments seen following the administration of an NO synthase inhibitor. Accordingly, a series of experiments were conducted to examine in detail whether impaired performance in the watermaze results from a specific effect on spatial learning or derives from action upon some other process (or processes) engaged in this and other kinds of tasks.

\section{Materials and Methods}

Subjects. Male hooded Lister rats $(250-450 \mathrm{gm} ; n=157)$ were used in all experiments. They were individually housed with ad libitum access to food and water. A $12 \mathrm{hr}$ light/dark cycle was maintained (0700-1900) with all testing carried out during the "light" phase. 
Apparatus. All behavioral testing was carried out in one of two openfield watermazes (Morris, 1981, 1984), each consisting of a large circular tank (diameter, $2.0 \mathrm{~m}$; depth, $0.6 \mathrm{~m}$ ) containing water at $25^{\circ} \mathrm{C}$ to a depth of $0.3 \mathrm{~m}$. The water was made opaque by the addition of powdered milk, which not only prevents the animals from seeing the platform but also allows efficient tracking of swim paths. In the spatial learning tasks, the rats were trained to find a hidden escape platform (diameter, $10 \mathrm{~cm}$ ) submerged $1-1.5 \mathrm{~cm}$ below the water surface. Both pools were in the center of a room containing various prominent cues (wall posters, wall cupboards, a rig of electrophysiological equipment, and a metal frame). The two rooms contained distinguishable extramaze cues. The paths taken by the animals in these pools were monitored by a video camera mounted in the ceiling. The resulting video signal was relayed to a video recorder, allowing both on- and off-line analysis, and from there to an image analyzer (HVS VP112). The $\mathrm{x}$ - and $\mathrm{y}$-coordinates of the rats' position were sampled at $10 \mathrm{~Hz}$ by an Archimedes computer (using a program called WATERMAZE) and stored on disk. This software can provide measures of latency, pathlength, swim speed, and so on, and the distribution of time spent in defined regions of the pool (e.g., quadrant, annulus from side wall, etc.). In the visual discrimination task, the rats were trained to escape onto one of two visible and distinguishable platforms (diameter, $10 \mathrm{~cm}$ ) whose visible surfaces were $1-1.5 \mathrm{~cm}$ above the water. One of these platforms (gray or black-and-white stripes) was rigid and provided escape from the water; the other (black-and-white stripes or gray) was floating and offered insufficient bouyancy to support a rat. A set of white curtains surrounded the pool occluding extramaze cues.

Behavioral pretraining. All rats (with the exception of those participating in the visual discrimination study) received $1 \mathrm{~d}$ of nonspatial pretraining consisting of six trials during which the extramaze cues were obscured from view by pulling the white curtains around the pool. The platform was moved randomly to a different position between trials. Rats that failed to find the platform after $60 \mathrm{sec}$ were guided to its location. The rats were allowed to stay on the platform for $30 \mathrm{sec}$ between trials. This pretraining is intended to allow the animals to become accustomed to the apparatus under conditions that prevent any spatial learning but allow them to learn that there is a means of escape from the water via the platform. Pretraining was conducted in the absence of any drug treatment, the day before the start of spatial training.

Drugs. L-NAME and L-arginine were obtained from Sigma, and D-NAME was obtained from Bachem. All drug solutions were made up in $0.9 \%$ physiological saline at an appropriate concentration such that an injection volume of $1 \mathrm{ml} / 100 \mathrm{gm}$ resulted in the final dosages listed below.

\section{Results}

Experiment 1: the effect of L-NAME on spatial reference memory (six trials per day)

The purpose of the following experiment was to examine whether L-NAME affects the acquisition of spatial reference memory in the watermaze using a standard training paradigm in which rats receive a block of six training trials on each day over a period of $3 \mathrm{~d}$. Additional groups of animals were also run to determine whether any deficit, resulting from L-NAME administration, was dose related, stereoselective, and/or reversible with coadministration of $L$ - arginine.

\section{Procedure}

Following $1 \mathrm{~d}$ of drug-free, nonspatial pretraining (trials 1-6), all animals $(n=53)$ were trained to find a fixed location, hidden escape platform ( $3 \mathrm{~d}$, six trials/day, ITI of 5-10 min, random start position across trials). Rats that failed to find the platform after $120 \mathrm{sec}$ were guided to its location. On each day, $1 \mathrm{hr}$ before the start of behavioral testing, they were injected intraperitoneally with either saline $(n=9), 10 \mathrm{mg} / \mathrm{kg} \mathrm{L-NAME}(n$ $=8), 75 \mathrm{mg} / \mathrm{kg} \mathrm{L}-\mathrm{NAME}(n=9), 75 \mathrm{mg} / \mathrm{kg}$ D-NAME $(n=9)$, $225 \mathrm{mg} / \mathrm{kg}$ L-arginine $(n=9)$, or $75 \mathrm{mg} / \mathrm{kg}$ L-NAME coadmin- istered with $225 \mathrm{mg} / \mathrm{kg}$ L-arginine $(n=9)$. Twenty-four hours after the final day of spatial training (and $1 \mathrm{hr}$ after injection), the rats' memory of the platform location was assessed in a transfer test during which the platform was removed from the pool and the rats allowed to swim freely for $60 \mathrm{sec}$.

Immediatcly following the transfer test, the rats reccived six additional "cue" trials during which they were required to escape onto to a visible gray platform (extending to $1-1.5 \mathrm{~cm}$ above the water surface). Both the platform location and the rats' start position were randomly changed from one trial to the next.

\section{Results}

All animals were capable of swimming around the pool until the platform was located and then climbing onto it. There was no visible sign of any sensorimotor disturbance either during swimming or in attempting to climb onto the platform. On the first day of spatial training, the $75 \mathrm{mg} / \mathrm{kg}$ L-NAME-treated animals appeared to be swimming faster than those in the other groups. An ANOVA of the mean swim speeds for each animal on the first day of spatial training revealed a significant difference $[F(5,47)=3.41 ; p<0.025]$ and subsequent Tukey's HSD pairwise comparisons indicated that only the $75 \mathrm{mg} / \mathrm{kg}$ L-NAME group (mean $=0.28 \pm 0.01 \mathrm{~m} / \mathrm{sec}$ ) differed significantly from the saline group (mean $=0.23 \pm 0.01 \mathrm{~m} / \mathrm{sec}$ ).

All animals showed a progressive decline in escape latency with training (Fig. 1A). An ANOVA of escape latencies for the $3 \mathrm{~d}$ of spatial training revealed an overall effect of group $[F(5,47)$ $=9.14 ; p<0.0001]$ and trial $[F(17,799)=28.54 ; p<0.0001]$, and a groups by trials interaction $[F(85,799)=1.36 ; p<0.05]$. Further analysis, using Tukey's HSD pairwise comparisons, showed that the $75 \mathrm{mg} / \mathrm{kg}$ L-NAME group performed significantly worse than the other groups $(p<0.01)$. Apart from the first trial, for which there was no difference in escape latency between groups $(F<1)$, this deficit was more pronounced over the early trials and, by the end of training, the $75 \mathrm{mg} / \mathrm{kg}$ L-NAME-treated rats were escaping the pool as quickly as the others, as reflected in the groups by trials interaction.

The rats' memory of the platform location was assessed in the transfer test (Fig. 1 $B, C$ ). All groups spent more time searching in the quadrant of the pool in which the platform had been located (the "training" quadrant), suggesting that the animals had learned something about the former location of the platform, but the groups differed with respect to the extent of learning [groups by quadrants interaction: $F(10,141)=2.34 ; p<$ $0.01]$. A second ANOVA of the percentage time spent in the training quadrant revealed only a significant effect of group $[F(5,47)=2.96 ; p<0.05]$, and subsequent Tukey's HSD pairwise comparisons showed that the $75 \mathrm{mg} / \mathrm{kg}$ L-NAME group was spending significantly less time in the training quadrant than the saline group $(p<0.05)$, and less time than that shown by the $10 \mathrm{mg} / \mathrm{kg}$ I,-NAMF, D-NAME, and the L-NAME + arginine groups considered together $(p<0.025)$. None of these three groups, or the L-arginine alone group, differed significantly from the saline-injected controls.

Immediately after the transfer test, the rats were given the "cue" task. All rats escaped from the pool rapidly (Fig. $1 D$ ) and there was no significant difference between the groups $(F<1)$.

The result of this first study is in agreement with Chapman et al. (1992) in demonstrating that L-NAME causes an impair- 


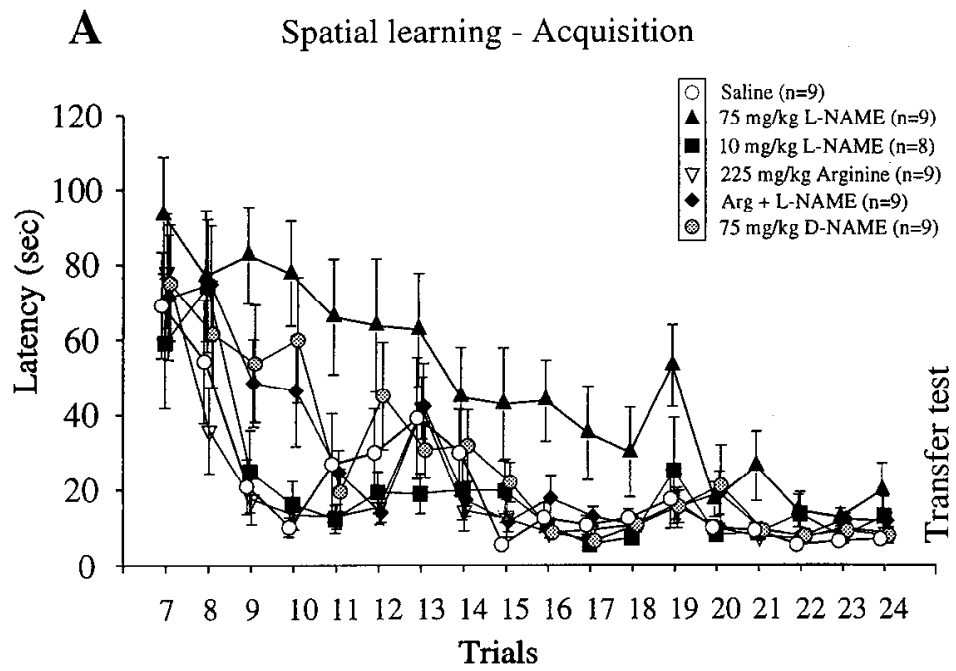

C

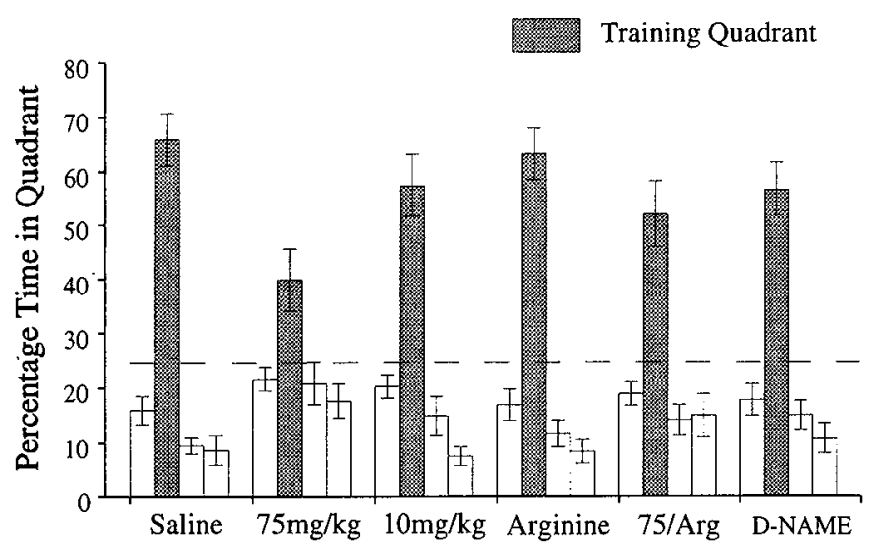

B Representative pathways during the transfer test

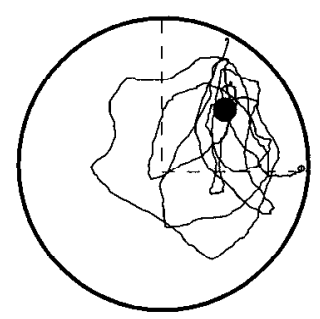

Saline

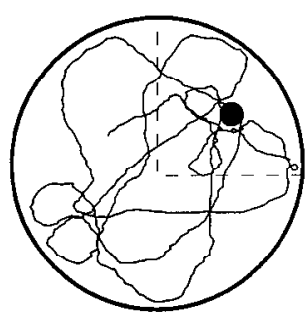

$75 \mathrm{mg} / \mathrm{kg}$ L-NAME
D

Cue task

Figure 1. Experiment 1. L-NAME causes an impairment of spatial reference memory (six trials per day). $A$, Mean escape latencies during acquisition. The $75 \mathrm{mg} / \mathrm{kg} \mathrm{L-NAME}$ group is impaired relative to saline-injected controls. $B$, Representative swimming paths taken during the transfer test (following 18 trials of spatial training). $C$, The mean percentage time spent in the four quadrants of the pool (organized with respect to the training quadrant) during the transfer test (trial 25). $D$, Mean escape latencies (of six trials) during the single visible platform task (cue task). There were no differences between the groups on this task.

ment in performance during the acquisition of a spatial reference memory task in the watermaze. This effect is dose related, stereoselective, and partially reversed by coadministration of $\mathrm{L}$-arginine. The absence of an effect of L-NAME on the cue task provides some evidence that the deficit in the spatial task is not due to some gross sensorimotor disturbance or alteration of motivational state.

Experiment 2: the effect of L-NAME on the retention of previously learned spatial information

The blockade of NMDA receptors with D-AP5 prevents the induction of LTP but has no effect on its expression or maintenance (Collingridge et al., 1983). If NMDA receptors are required to initiate changes in synaptic weights in the hippocampus, but not to maintain a specific distribution of synaptic weights, then one might predict that D-AP5 would have no effect on the retrieval of spatial information despite, at the same time, im- pairing new learning. Behavioral experiments have shown that the intracerebral infusion of D-AP5, at concentrations sufficient to block the induction of LTP in vivo, does not affect the retrieval of a previously acquired platform location in the watermaze (Morris et al., 1990). However, in a parallel study, the same dose of D-AP5 did impair learning about a novel platform location in the same spatial environment.

The precise role, if any, of NO in LTP is not yet fully understood. Evidence from in vitro studies suggests that NO could be involved in initial induction processes but not the long-term expression of potentiation. Haley et al. (1992) demonstrated that L-NAME did not affect synaptic responses evoked by lowfrequency stimulation either before or after LTP induction. In addition, Schuman and Madison (1991) reported that inhibition of LTP by L-methyl arginine was not reversed by L-arginine applied post-tetanically but was prevented by coadministration during the tetanus. If the expression of LTP requires the con- 
A

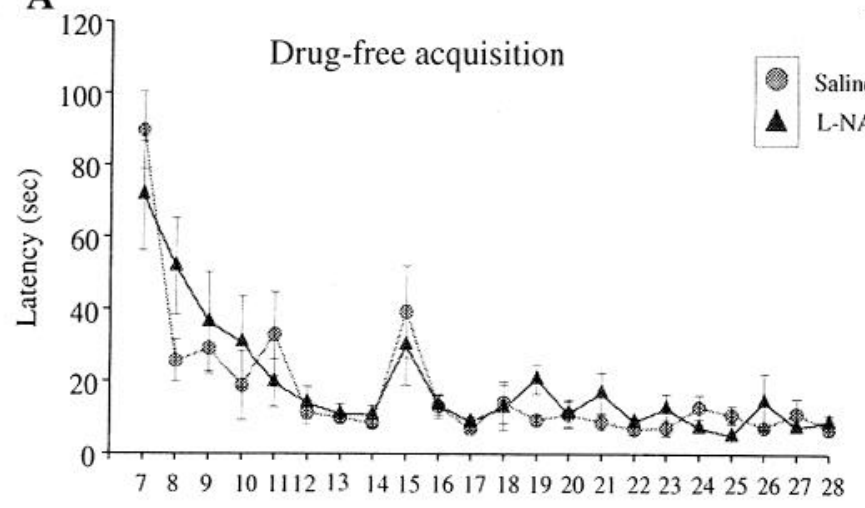

B



Trial no.

C Transfer test 1

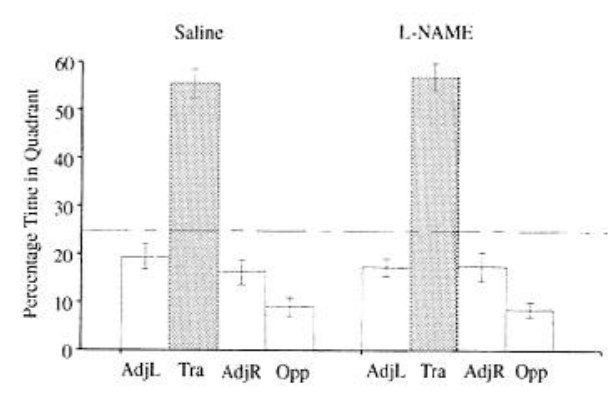

Transfer test 2

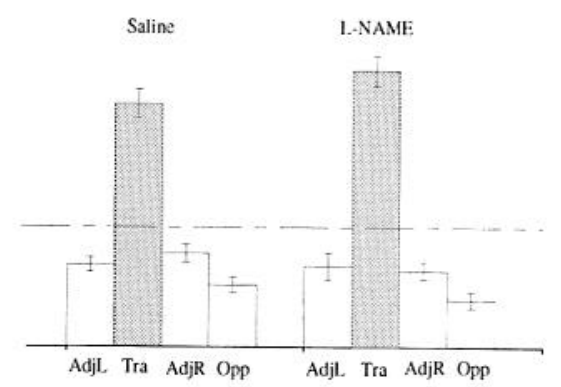

Figure 2. Experiment 2. L-NAME $(75 \mathrm{mg} / \mathrm{kg})$ does not affect the retention of previously learned spatial information. $A$, Mean escape latencies during the initial, drug-free, spatial training. $B$, Mean escape latencies during retention. $C$, The mean percentage time spent in the four quadrants of the pool (organized with respect to the training quadrant) during the first transfer test after four retention trials (trial 33). $D$, Transfer test 2 after eight retention trials (trial 38).

tinued, persistent activation of NO synthase, then the addition of $\mathrm{L}$-arginine should reverse the enzyme blockade and result in the immediate expression of LTP. The fact that arginine only reverses the L-methyl arginine inhibition of LTP if present during the tetanic stimulation supports the idea that NO is involved in induction but not long-term expression. Accordingly, if LTP is a substrate for spatial learning, and if NO is involved in LTP, then one might predict that NO synthase inhibition would prevent new learning but spare retention, in a manner parallel to the behavioral results obtained with D-AP5.

\section{Procedure}

Following $1 \mathrm{~d}$ of nonspatial pretraining (trials 1-6), rats $(n=$ 20) were extensively trained (in the absence of any drug injection) to find a fixed location, hidden escape platform ( $3 \mathrm{~d}$ of four trials/day, $3 \mathrm{~d}$ of two trials/day, and $4 \mathrm{~d}$ of one trial/day; trials 7-28). Following a $3 \mathrm{~d}$ interval (in order to equate the training protocol with that used in the D-AP5 experiment of Morris et al., 1990), they then received another $8 \mathrm{~d}$ of training with one trial per day to the same platform location. At this stage, the rats were injected with saline $(n=10)$ or L-NAME $(75 \mathrm{mg} / \mathrm{kg} ; n=10)$ each day, $1 \mathrm{hr}$ prior to the start of behavioral testing. Performance was assessed with a first transfer test, 24 hr after trial 32 (and immediately before trial 34), and again 24 hr after trial 37.

\section{Results}

During the initial, drug-free, spatial training, all animals learned something about the the location of the hidden platform as indicated by direct swim paths and short escape latencies on the later training trials (Fig. 2A). On the first trial after drug administration (trial 29), both groups of animals showed good retention of the platform location and escaped rapidly from the water with no significant difference in terms of escape latency $[F(1,18)=1.39 ; p=0.25]$. All rats continued to escape rapidly from the pool during the eight training trials of the drug phase (Fig. $2 B)$. An ANOVA revealed no effect of group $[F(1,18)=$ $1.10 ; p=0.31]$ or trial $[F(7,126)=1.04 ; p=0.41]$, nor a groups by trials interaction $[F(7,126)=1.04 ; p=0.41]$. The two transfer tests (trials 33 and 38; Fig. 2C,D) also showed that both groups searched persistently in the training quadrant. An ANOVA of performance in the first transfer test showed a significant effect of quadrant $[F(2,54)=105.81 ; p<0.001]$ but no groups by quadrants interaction $(F<1)$. Similar results were obtained in the second transfer test [quadrants $F(2,54)=111.59 ; p<0.001$; groups by quadrants interaction $F(2,54)=1.73 ; p>0.10$ ]. 
A

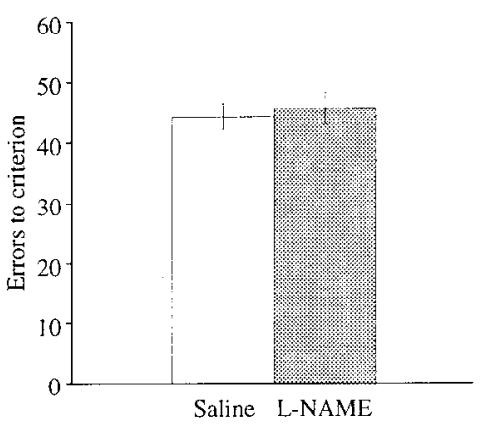

B

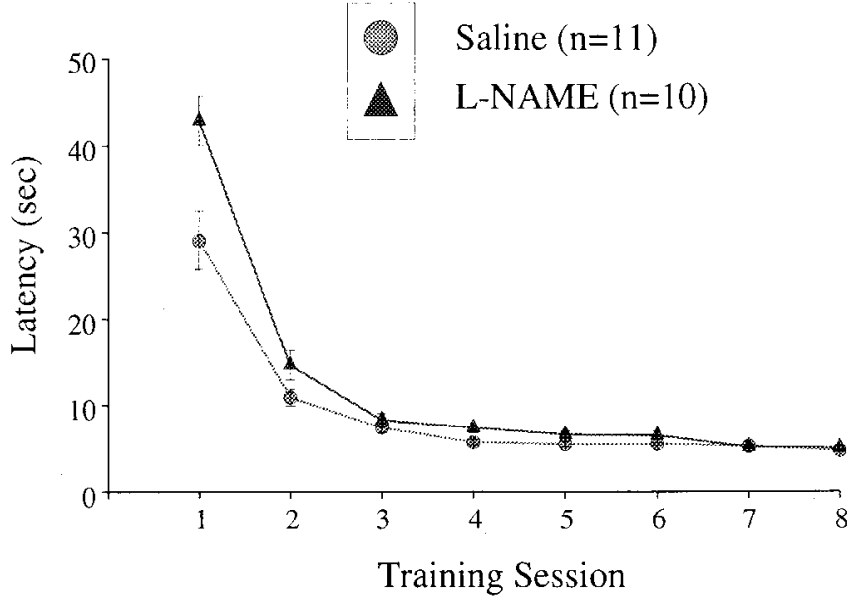

Figure 3. Experiment 3. The effect of L-NAME on a two platform, visual discrimination task in the watermaze. $A$, The number of errors made in attaining a criterion of 18 of 20 correct choices. L-NAME $(75 \mathrm{mg} / \mathrm{kg})$ does not impair the acquisition of the visual discrimination. $B$, Mean escape latencies during the first eight training sessions. L-NAME-treated rats took significantly longer to escape from the pool on the first training session.

In summary, L-NAME does not affect the retention of previously acquired spatial information. This result is also in agreement with that reported by Chapman et al. (1992).

\section{Experiment 3: the effect of L-NAME upon visual discrimination learning in the watermaze}

It is possible that the L-NAME induced deficit in experiment 1 is due to an action of the drug on some process other than spatial learning. For example, L-NAME may impair performance through an action on some procedural component of the task. However, no impairment was seen in the cue task and we can therefore make a limited claim, namely, that the animals do not exhibit any gross sensorimotor disturbance that prevents them from swimming freely, or seeing and then climbing onto the platform. In addition, the fact that L-NAME has no effect on retention of spatial information further supports this interpretation. A more compelling claim for a specific effect of $\mathrm{NO}$ synthase inhibition on the neural mechanisms of spatial learning could be made if the inhibitor had no detectable effect on visual discrimination learning in the watermaze. Visual discrimination learning does not require the integrity of the hippocampal formation (O'Keefe and Nadel, 1978; Morris et al., 1986a) and is unimpaired by the intracerebroventricular infusion of D-AP5 (Morris et al., 1986b). The fact that this form of learning would appear to occur independently of NMDA receptor-mediated synaptic plasticity provides one suitable control task. If the L-NAME-induced deficit in the spatial task is due to a blockade of NMDA receptor-mediated synaptic plasticity, then L-NAME should be without effect on the visual discrimination task. On the other hand, a nonspecific action of the drug might be expected to have a detrimental effect on performance in both the spatial and nonspatial tasks.

\section{Procedure}

Separate groups of rats were trained to discriminate between a rigid and a floating visible platform. Injections of L-NAME (75 $\mathrm{mg} / \mathrm{kg} ; n=10$ ) or saline ( $n=11$ ) were given daily $1 \mathrm{hr}$ before testing (as in experiment 1). Rats received 10 trials per day, with an ITI of 5-10 min, until a criterion of $90 \%$ correct choices had been achieved over 2 consecutive days ( 18 of 20). On each trial, the rat was placed into the water facing the side wall at a point across the pool from the two visible platforms; these were moved randomly around the pool between trials in order to vary the spatial location of the rewarded platform. In addition, a pseudorandom sequence was used to alternate the left/right orientation of the rewarded/nonrewarded platforms. In a final test conducted the day after reaching criterion, the rats were presented with two visibly identical platforms, only one of which was rigid and a means of escaping the water. If the rats were attending to the visual appearance of the platforms to solve the task, then performance should fall to chance.

\section{Results}

After several days of training, the animals began to make a deliberate choice for one platform or the other. By day 8 , they were starting to achieve the criterion level of performance and all had reached criterion by day 17. An ANOVA of choice accuracy over the first eight training sessions revealed no differences between the groups $(F<1)$, nor any groups by sessions interaction $[F(7,133)=1.25 ; p=0.28]$. In addition, there was no difference between the groups in terms of the number of errors made in achieving criterion (saline, $44.3 \pm 2.2$; L-NAME, $45.6 \pm 2.6 ; F<1$; Fig. $3 A$ ). Performance fell to chance on the day of training with two identical platforms $(52.9 \pm 2.3 \%)$.

Closer inspection of the first day of training, however, revealed differences between the saline-treated and L-NAMEtreated groups. The L-NAME group took significantly longer to escape from the pool on the early training trials (Fig. 3B). An ANOVA of the mean escape latencies for the first eight training sessions revealed a significant effect of group $[F(1,19)=10.01$; $p<0.005]$ and session $[F(7,133)=156.75 ; p<0.0001]$, and a groups by sessions interaction $[F(7,133)=6.90 ; p<0.0001]$. A subsequent analysis of simple main effects showed that the two groups differed significantly on day $1(F=55.68 ; p<0.001)$. On day 2, there was still a trend toward a difference between the groups $(F=2.95 ; p=0.09)$, but the escape latencies of the 


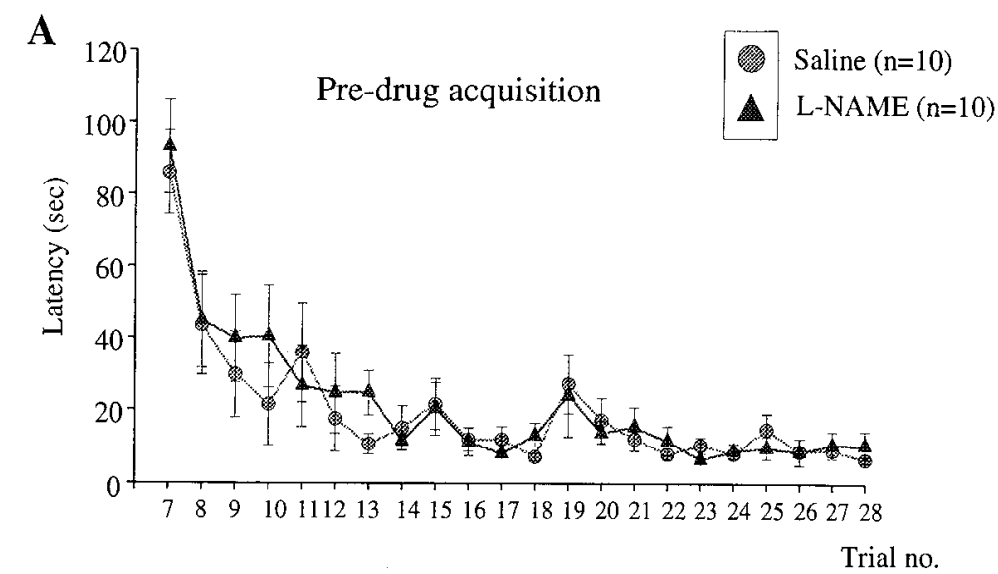

B

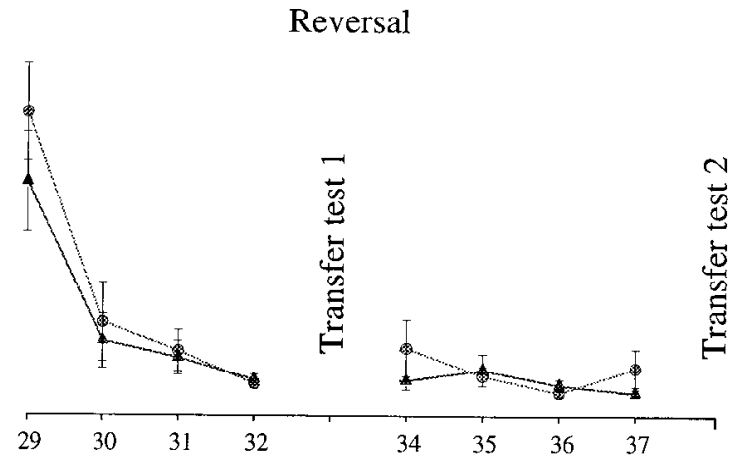

C

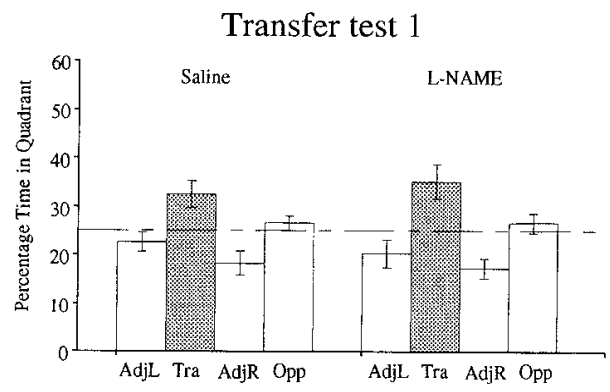

D

Transfer test 2

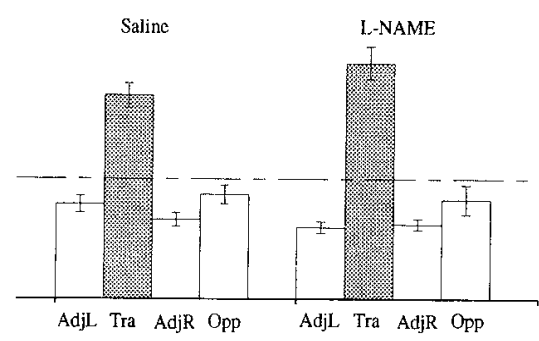

Figure 4. Experiment 4. L-NAME does not impair the acquisition of a novel platform location in a familiar spatial environment (reversal). $A$, Mean escape latencies during the initial, drug-free, spatial training. $B$, Mean escape latencies during reversal. L-NAME (75 mg/kg) did not impair the acquisition of a novel platform location in a familiar spatial environment. $C$, The mean percentage time spent in the four quadrants of the pool (organized with respect to the training quadrant) during the first transfer test after four reversal trials (trial 33). $D$, Transfer test 2 after eight reversal trials (trial 38).

two groups were indistinguishable on subsequent training sessions. The basis for the longer escape latencies on day 1 is unclear, but a contribution may be that the L-NAME-treated animals showed a greater tendency than saline-treated animals toward failing to escape from the water at all within $60 \mathrm{scc}$ $[F(1,19)=4.05 ; p=0.06]$.

L-NAME did not affect the rate at which criterion levels of performance were attained in the visual discrimination task. There was, however, a subtle effect of the inhibitor on performance on the first day of training that resulted in prolonged escape latencies and a tendency to fail to climb onto the stable platform within $60 \mathrm{sec}$. This apparent "first day" effect of L-NAME in the watermaze could be of more consequence in a more rapidly acquired task such as the spatial reference memory task, and thus may contribute toward the deficit observed in experiment 1 .

Experiment 4: the effect of L-NAME on acquisition of a novel platform location in a familiar spatial environment

The results of experiments 1-3 suggest a profile of impairment similar to that reported to occur following chronic intraventricular infusion of AP5: an impairment of spatial learning without effect upon retention or visual discrimination learning (Morris et al., 1986b, 1990). However, the strikingly longer escape latencies of the L-NAME group during day 1 of the visual dis- crimination task points to a different possibility. Specifically, L-NAME may cause a transient and nonspecific impairment that can affect performance in several different types of learning, rather than a direct effect on the process of spatial learning per sc.

A series of experiments was conducted to distinguish these two possibilities. The first of these, run in parallel with experiment 2, began with the same drug-free initial training but, during the drug phase of the experiment, the platform was moved to the opposite quadrant of the pool (a type of "reversal" task). As previously mentioned, D-AP5 has been reported to impair performance in such a reversal task, presumably because new learning is involved (Morris et al., 1990).

\section{Procedure}

Separate groups of rats wcre trained as in experiment $2(n=10$ per group), with the exception that, during the drug phase, the platform was moved to the opposite quadrant of the pool (i.e., rats trained initially to $\mathrm{SW}$ had the platform moved to NE).

\section{Results}

As in experiment 2, all animals demonstrated a considerable reduction in escape latencies during initial training (Fig. 4A). On the first trial during the drug administration phase (trial 29), 
$\boldsymbol{\Lambda}$

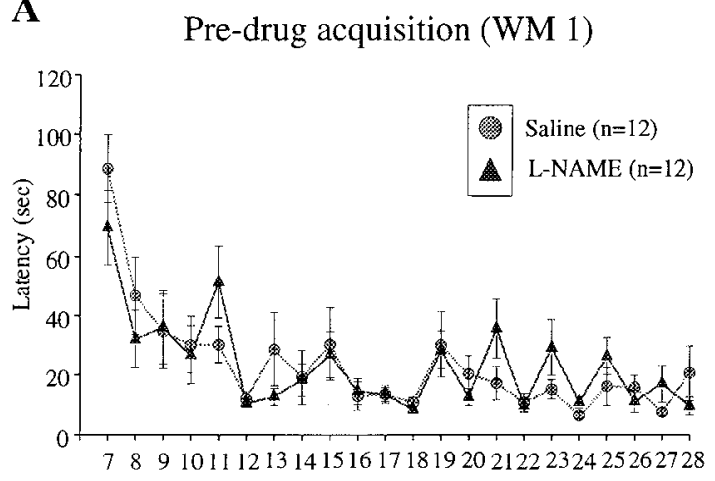

D

\author{
Drug-phase acquisition (WM 2)
}

Trial no.

B

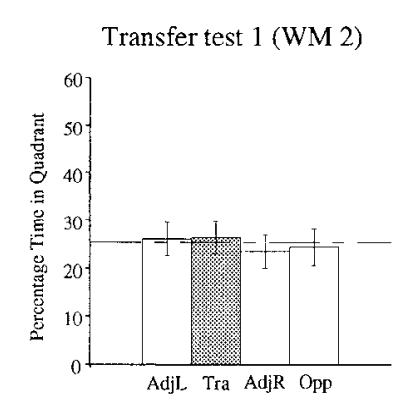

C

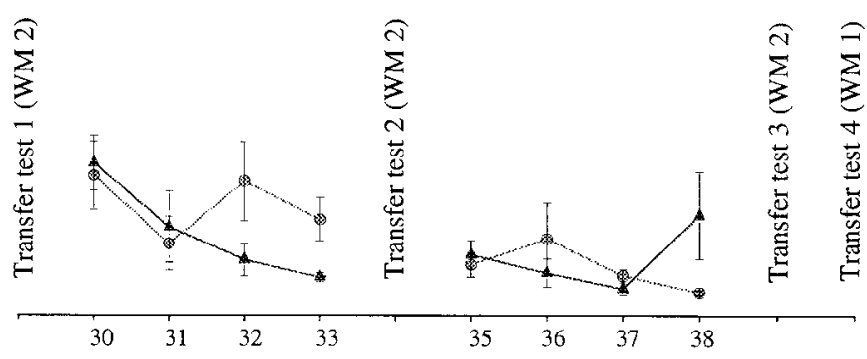

Trial no.

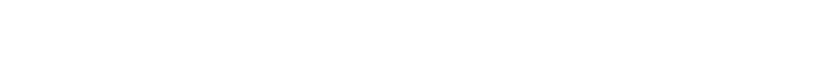


more time in the new location and, thus, their escape latencies reduced across trials (Fig. $4 B$ ). An ANOVA revealed a significant improvement across trials $[F(7,126)=21.02 ; p<0.0001]$, but no effect of group $[F(1,18)=2.17 ; p=0.16]$ nor any groups by trials interaction $(F<1)$. Transfer tests conducted $24 \mathrm{hr}$ after trials 32 and 37 also showed that L-NAME had no effect on the acquisition of the new platform location (Fig. 4C,D). An ANOVA of the first transfer test revealed a significant effect of quadrant $[F(2,54)=12.60 ; p<0.001]$, but no groups by quadrants interaction $(F<1)$. Similarly, on the second transfer test, there was a significant effect of quadrant $[F(2,54)=59.93 ; p<0.001]$ but again no interaction with the drug treatment $[F(2,54)=$ $1.83 ; p>0.10]$. Therefore, and somewhat surprisingly, L-NAME did not impair the learning of a novel platform location in a familiar spatial environment.

Experiment 5: the effect of L-NAME on spatial learning in a novel environment by experienced animals previously trained in another watermaze

The lack of an effect of L-NAME on the "reversal" task (experiment 4) may be explained in two ways. First, the amount of new lcarning required to solve the "reversal" task may be very small. During the initial drug-free phase, a relatively detailed representation of the environment will already have been formed and so, when the platform is moved, the only modification required of that representation is to encode the new goal location. There is no need to "reverse" the learned spatial relationships between extramaze cues. Learning a new goal location in a familiar environment may necessitate very little, if any, synaptic plasticity. Alternatively, it is possible that the L-NAME-induced deficit in experiment 1 is, as noted above, due to an action of the drug on some process other than spatial learning which detrimentally affects performance early in training. To distinguish these two possibilities, a further experiment was conducted in which the initial watermaze training was carried out in a different spatial environment from that used during the drug phase (Gallagher, 1985; Shapiro and O'Connor, 1992). With this training protocol, the experienced animals then have to learn about a wholly novel spatial environment during the drug phase, in contrast to the "reversal" task in which they have only to learn a new platform location. In this experiment, the training protocol was otherwise identical to that used in the retention/reversal experiments (experiments 2 and 4).

\section{Procedure}

Following $1 \mathrm{~d}$ of nonspatial pretraining (trials 1-6), the rats ( $n$ $=24$ ) were given spatial training in watermaze 1 ( $3 \mathrm{~d}$ of four trials/day, $3 \mathrm{~d}$ of two trials/day, and $4 \mathrm{~d}$ of one trial/day; trials 7-28). Following a $3 \mathrm{~d}$ interval (in order to equate the training protocol to that used in experiments 2 and 4), a subgroup of eight rats (made up of four animals from each of the saline and L-NAME groups) was given a transfer test in watermaze 2 (trial 29 ) in order to assess whether training in watermaze 1 resulted in a spatial bias towards any one quadrant in watermaze 2. Immediately following this test, these and the remaining rats began their eight trials of spatial training in watermaze 2 ( $1 \mathrm{trial} /$ day), being injected with saline $(n=12)$ or L-NAME $(75 \mathrm{mg} /$ $\mathrm{kg} ; n=12$ ) $1 \mathrm{hr}$ prior to the start of behavioral testing each day. In watermaze 1 , the platform was located in one of the four quadrants of the pool. In watermaze 2 , the platform was located in either the NE or SW quadrant. For every four rats trained to a particular platform position in watermaze 2 , each of the four platform positions had been used during training in watermaze 1. Assignment of rats to groups was counterbalanced with respect to the platform position in both watermazes 1 and 2 and performance over the last four training trials in watermaze 1 (trials 25-28). Performance in watermaze 2 was assessed with respect to escape latency and using a transfer test, $24 \mathrm{hr}$ after trial 33 (and immediately before trial 35) and again $24 \mathrm{hr}$ after trial 38. Finally, the rats were returned to watermaze 1 and retention of the platform location was assessed with a transfer test in the absence of any drug injection (trial 40).

\section{Results}

During spatial training in watermaze 1 , both groups of animals learned to escape from the water rapidly. An ANOVA of escape latencies revealed a highly significant improvement across trials $[F(21,462)=10.42 ; p<0.0001$; Fig. $5 A)$.

The first transfer test in watermaze 2 (trial 29), before the start of spatial training, was analyzed in two ways. First, the data was expressed in terms of the "future" training quadrant (Fig. 5B). An ANOVA showed no significant bias to any one quadrant $(F<1)$. Second, the data was reanalyzed in terms of the actual quadrants described in terms of compass directions (Fig. 5C); this showed a small bias toward the NW and SW quadrants $[F(2,21)=4.43 ; p<0.025]$. This corresponds to a bias toward the door into the watermaze room and the location of the carrying cage.

The rats learned about the new spatial environment extremely rapidly, showing a mean escape latency of less than $20 \mathrm{sec}$ by trial 4. An ANOVA of the escape latencies from the eight training trials in watermaze 2 (Fig. $5 D$ ) revealed that there was a significant improvement across trials $[F(7,54)=3.87 ; p<0.001]$ but no overall effect of group $[F<1]$. There was, however, a just significant groups by trials interaction $[F(7,54)=2.10 ; p=$ $0.05]$. A subsequent analysis of simple main effects revealed group differences on trials 32 and $38(p<0.05)$. However, the fact that on trial 32 the saline group was taking, on average, longer to escape, while on trial 38 the opposite was true, suggests that these differences are most likely due to random fluctuations in performance rather than to some substantive underlying cause.

An ANOVA of the transfer test (trial 34; Fig. $5 E$ ), conducted after four training trials in watermaze 2 , revealed a strong bias toward the training quadrant $[F(2,66)=18.13 ; p<0.001]$. There was no groups by quadrants interaction $(F<1)$. This result was repeated in the second transfer test [trial 39 ; Fig. $5 F$; $F(2,66)=78.34 ; p<0.001]$, with there again being no interaction with drug treatment $(F<1)$.

Finally, when the rats were returned to watermaze 1 , both groups still showed a significant and equivalent bias toward the appropriate training quadrant reflecting their memory of the original training environment despite the intervening training (trial 40; Fig. 5G). An ANOVA showed a significant effect of quadrant $[F(2,66)=9.01 ; p<0.001]$ with no groups by quadrants interaction $(F<1)$.

In summary, L-NAME did not impair the acquisition of spatial reference memory in experienced animals that had previously been trained on a watermaze task in a different spatial environment.

\section{Experiment 6: the effect of L-NAME on spatial reference memory (one trial per day)}

Examining the pattern of results obtained so far, it is possible that L-NAME only impairs performance early in training in 
A
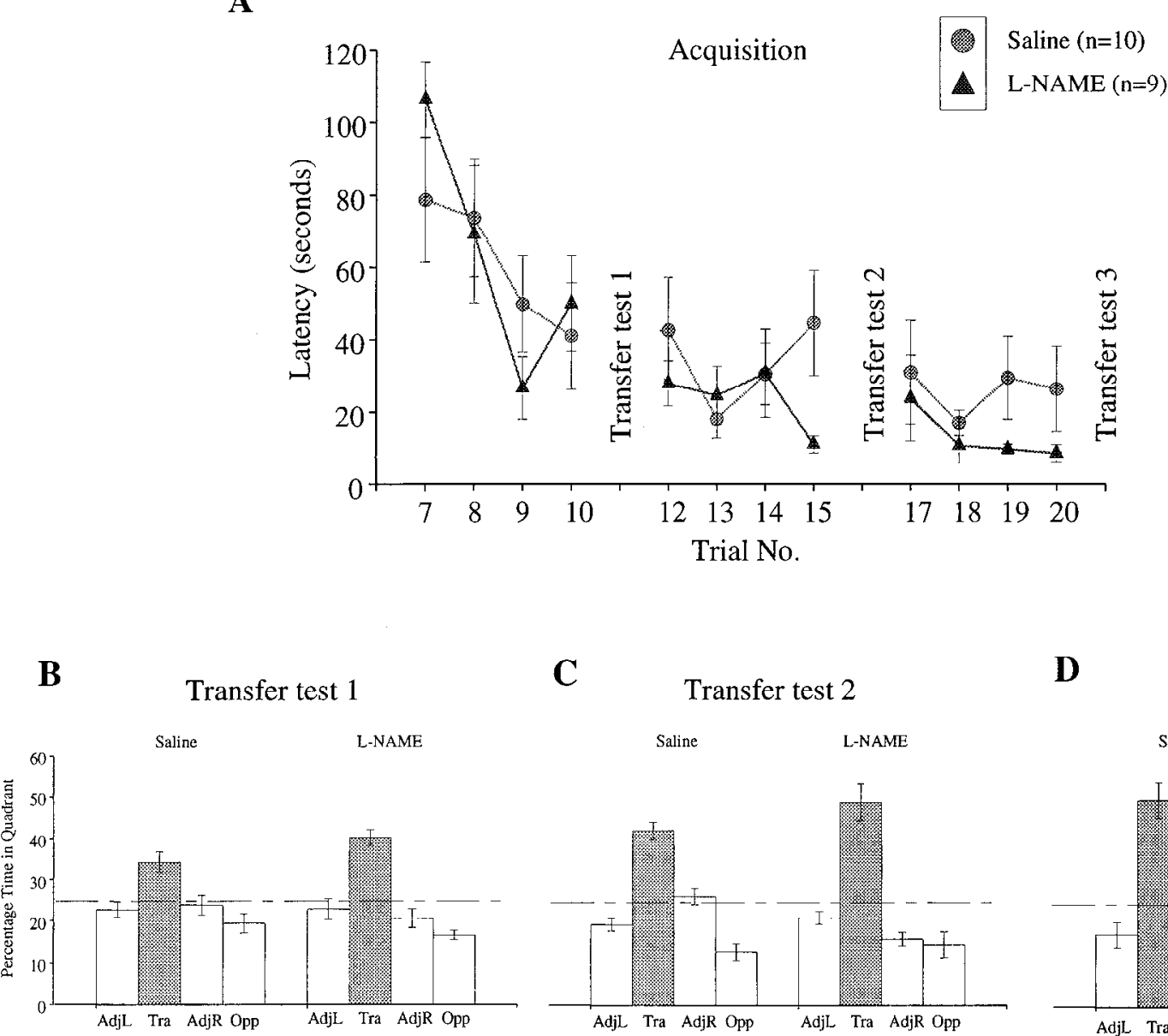

C

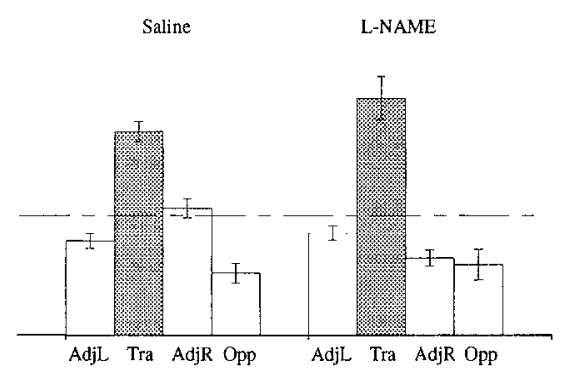

D

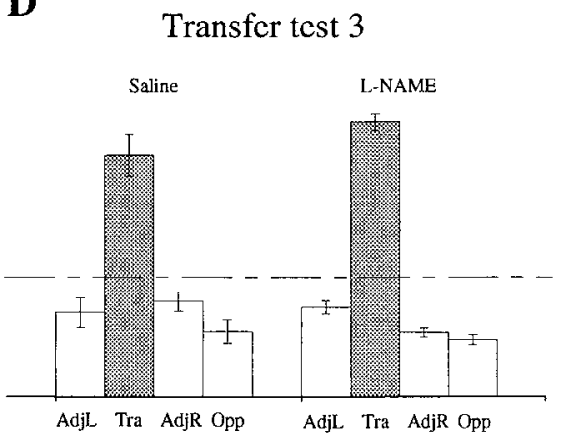

Figure 6. Experiment 6. L-NAME does not affect spatial reference memory (one trial per day). $A$, Mean escape latencies during acquisition. $B$, The mean percentage time spent in the four quadrants of the pool (organized with respect to the training quadrant) during the first transfer test after four spatial training trials (trial 11). C. Transfer test 2 after eight spatial training trials (trial 16). $D$, Transfer test 3 after 12 spatial training trials (trial 21).

tasks (spatial and visual discrimination) that involve the animals receiving multiple trials with a short intertrial interval (ITI). L-NAME treatment resulted in a deficit in a spatial reference memory task in which the animals received a block of six trials per day with an ITI of 5-10 min (experiment 1). A difference between L-NAME and saline groups was also seen on the first day of training in the visual discrimination task (10 trials per day, ITI $=$ 5-10 min; experiment 3 ). In contrast, the performance of the L-NAME animals was indistinguishable from saline controls in tasks in which there was only one trial per day (experiments 2, 4, and 5). An alternative possibility is that the lack of a drug effect in experiments 2,4 , and 5 is due to the animals being experienced in the watermaze prior to training under the influence of the drug. The following experiment was therefore conducted to investigate whether L-NAME would affect spatial reference memory in naive animals when only a single training trial was given each day.

\section{Procedure}

Following $1 \mathrm{~d}$ of nonspatial pretraining (trials 1-6), spatial reference memory was assessed in experimentally naive rats $(n=$ 19) that were given only one spatial training trial per day for a total of $12 \mathrm{~d}$. Performance of salinc- $(n=10)$ and L-NAME$(75 \mathrm{mg} / \mathrm{kg} ; n=9)$ injected rats was assessed in terms of escape latency and by means of three transfer tests, $24 \mathrm{hr}$ after trial 10 (and immediately before trial 12), $24 \mathrm{hr}$ after trial 15 (and immediately before trial 17) and, finally, $24 \mathrm{hr}$ after trial 20.

\section{Results}

An ANOVA of the escape latencies showed a significant improvement across the 12 training trials $[F(11,187)=8.81 ; p<$ 0.0001 ; Fig. $6 A$ ). However, there was no significant effect of group $(F<1)$, nor any groups by trials interaction $[F(11,187)$ $=1.07 ; p=0.39]$. L-NAME did not affect the amount of time spent in the training quadrant during the transfer tests. An ANOVA of transfer test 1 (trial 11; Fig. 6B) revealed a significant effect of quadrant $[F(2,51)=22.08 ; p<0.001]$ but no groups by quadrants interaction $[F(2,51)=1.42 ; p>0.20]$. Analysis of transfer test 2 (trial 16; Fig. $6 C$ ) showed both an effect of quadrant $[F(2,51)=49.54 ; p<0.001]$ and an interaction with drug treatment $[F(2,51)=3.35 ; p<0.05]$. However, a second ANOVA of the time spent solely in the training quadrant did not reveal a difference between the groups $[F(1,17)=2.09 ; p=$ 0.17 ]. An ANOVA of transfer test 3 (trial 21; Fig. 6D) also revealed a significant effect of quadrant $[F(2,51)=90.04 ; p<$ $0.001]$ but no interaction with drug treatment $[F(2,51)=1.90$; $p>0.10]$. It appcars, thcrefore, that L-NAME trcatment does not impair spatial reference memory in the watermaze if the 
animals receive only one training trial per day. This lack of effect is, statistically, neither a "ceiling" nor a "floor" effect in that both groups showed a progressive increase in the bias toward the training quadrant over the three transfer tests.

\section{Discussion}

The main findings of this series of experiments were that animals injected intraperitoneally with a dose of L-NAME sufficient to bring about a $>90 \%$ reduction in hippocampal NO synthase (Bannerman et al., 1994) showed (1) impaired performance in both a spatial reference memory and a visual discrimination task on early training trials (when these were conducted with multiple trials per session), and (2) that the same dose of L-NAME was without effect on performance in retention, reversal learning, the learning of a new spatial environment in watermazeexperienced animals and, even, acquisition of the basic watermaze task in experimentally naive animals trained at one trial per day.

\section{Does L-NAME impair spatial learning?}

These results are consistent with previously published reports on the effects of systemic NO synthase inhibitors on learning in the watermaze (Chapman et al., 1992). L-NAME impairs the acquisition of a spatial reference memory task with multiple trials per session but does not affect the retention of previously learned spatial information. This impairment is dose related, stereoselective, and reversed by coadministration of L-arginine. In an attempt to determine the behavioral specificity of this impairment, performance in a visual discrimination task was assessed. The rate at which criterion levels of performance were attained was unaffected by L-NAME. This apparent dissociation between the effects of L-NAME on performance of a spatial task and a visual discrimination task is, in several respects, similar to that obtained with D-AP5 (Morris et al., 1986b), and with selective hippocampal lesions (Morris et al., 1986a). This suggests that L-NAME-treated rats do not exhibit a gross sensorimotor impairment and can indeed learn some tasks normally.

Closer analysis of the visual discrimination task revealed, however, that the L-NAME animals took significantly longer to escape from the pool during the first training session. This deficit prompted the suggestion that L-NAME causes a transient but more general disturbance of function extending beyond the domain of spatial learning. Irrespective of the nature of this disturbance, it could also account for the apparent impairment of spatial learning in experiment 1 , bearing in mind that the 18 trials of training took place over only $3 \mathrm{~d}$ and the deficit appears to be largest over the first $2 \mathrm{~d}$. If L-NAME were, on the other hand, actually disrupting the mechanisms underlying spatial learning, then rats should be impaired relative to controls without regard to intertrial interval or the number of trials per session. The results of the platform reversal task (experiment 4) and the two watermaze tasks (experiment 5) raises problems for this hypothesis and suggests that the initial deficit (cxpcriment 1) is due to a subtle but less specific effect. The result of the one trial/day spatial reference memory task (experiment 6) further calls into question the hypothesis that L-NAME is disrupting spatial learning. Clearly, we cannot rule out the possibility that a more complete inhibition of NO synthase may result in a real impairment of spatial learning. Gene knockouts of brain NO synthase may provide an opportunity to realize more complete inhibition (Huang et al., 1993).
What are the implications for the relationship between LTP and learning?

Given the fact that NO synthase inhibitors block the induction of LTP in vitro, our interpretation of the behavioral results would appear to contradict the hypothesis that LTP and certain kinds of learning share a common underlying mechanism. This is not so: in a parallel study (following article, Bannerman et al., 1994), we show that the very same dose and route of administration of L-NAME (causing a $>90 \%$ inhibition of NO synthase whether given acutely or chronically) does not appear to block the induction of NMDA-dependent LTP in the dentate gyrus in vivo. Thus, an accurate description of the two studies is that a compound that does not block LTP does not impair spatial learning. This result does not contradict the hypothesis of a link between LTP and learning. L-NAME did, however, cause a number of physiological and behavioral changes - a reduction in dentate field potentials, cerebrovascular changes, and a transient behavioral dysfunction affecting both a hippocampal-independent and a hippocampal-dependent task.

\section{What is the nature of the functional disturbance induced by $L-N A M E$ ?}

In attempting to provide an explanation for the observed pattern of results, two distinct but related questions need to be addressed. First, what is it about the various tasks used in this study that results in impaired performance in some, but not other, paradigms? Second, what possible physiological actions of L-NAME may be responsible for the effects on performance? It may then be possible to determine how these two factors interact to produce the observed pattern of results.

Why is impaired performance seen in some, but not other, paradigms? L-NAME impairs performance in tasks involving training with multiple trials and short ITIs. In contrast, L-NAME-treated rats receiving just one trial/day are unimpaired. One possibility is that the physical demands of a task involving multiple trials are much greater and that animals become tired following repeated swims. Drug treatment may accentuate this fatigue and this, in turn, could indirectly interfere with the animals' ability to learn. This account predicts a deficit in both the spatial and the visual discrimination tasks for as long as the animals are taking a considerable time to escape from the water. As performance improves, however, the druginduced deficit would be expected to disappear-as it did in both experiment 1 and 3 . Additionally, there are several potential differences at a psychological level between the multipleand single-trial paradigms. With one trial/day, the rats find the platform and are then removed to their home cage for the rest of the day - a satisfactory escape. In contrast, with multiple trials, having first found the platform, the rats are soon after put back into the water for further trials. Thus, the platform may be less rewarding with multiple trials offering lesser incentive to escape. Repeated sequential exposure to the aversive elements of the watcrmaze in the multiple trial paradigm may, quite separately, be more anxiogenic. L-NAME treatment could conceivably interact with the altered psychological state of the animal during multiple-trial paradigms in such a way as to interfere, albeit indirectly, with the ability to learn. In the singletrial paradigms, however, locating the platform results in a complete escape from the water for $24 \mathrm{hr}$, which could potentially make the task less stressful. Similarly, as animals become more familiar with the procedural demands of the task during training, 
they become less anxious. In more general terms, any druginduced feeling of ill-being could conceivably interact with physiological factors such as fatigue or psychological factors such as motivation or anxiety to produce an apparent learning impairment. Neither account requires that L-NAME interacts with, or that NO is involved in, the neural mechanisms underlying the associative processes of spatial or visual discrimination learning.

What physiological actions of L-NAME may be responsible for the impairments in performance? There is a growing literature regarding putative roles of NO throughout the body including effects on the vasculature, immune system, viscera, and spinal chord (Garthwaite, 1991; Moncada, 1992; Schuman and Madison, 1993). In addition, the NO synthase enzyme is found in many brain areas (Bredt et al., 1991; Vincent and Kimura, 1992) and there are numerous reported actions of NO synthase inhibitors in a wide variety of brain regions. The use of an intraperitoneal injection as the route of administration is likely to result in a near global inhibition of NO synthase throughout the body. For example, in the parallel physiological study (Bannerman et al., 1994), there is evidence for a near complete enzyme inhibition in three brain areas (cortex, cerebellum, and hippocampus), a global cerebral oligemia, and a dramatic rise in blood pressure. There is also a decrease in the size of the hippocampal field potentials and it is possible that similar reductions in function may occur in other brain areas.

There are, therefore, numerous potential sites of action for L-NAME following an intraperitoneal injection, and it is not known how a physiological effect of this inhibitor may interact with the different physical and psychological demands of the tasks we have used. We cannot rule out the possibility that a rapid onset tolerance develops, which could account for the fact that there is a deficit on the early training sessions (experiments 1 and 3 ) that then disappears. At the molecular level, this seems unlikely because both we (Bannerman et al., 1994) and Dwyer et al. (1991) have shown that the level of NO synthase inhibition actually increases with repeated injections. At the psychological level, however, a tolerance to the effects of the drug remains possible. In addition, we cannot rule out the possibility that NO synthase inhibition may have a direct effect upon the learning mechanisms involved in other tasks (e.g., conditioned eyeblink responding, Chapman et al., 1992; social interaction, Böhme et al., 1993; one trial inhibitory avoidance, Hölscher and Rose, 1992).

To conclude, L-NAME causes a behavioral syndrome that is likely to extend beyond the domain of learning per se. Furthermore, intraperitoneal injection of L-NAME results in a multiplicity of physiological actions in vivo that does not include a block of LTP. These findings neither compromise nor support the hypothesis that the mechanisms of LTP underly certain types of learning, but point to the need for further enquiry into the functional role of $\mathrm{NO}$ in the brain.

\section{References}

Bannerman DM, Chapman PF, Kelly PAT, Butcher SP, Morris RGM (1994) Inhibition of nitric oxide synthase does not prevent the induction of long-term potentiation in vivo. J Neurosci 14:7415-7425.

Bliss TVP, Collingridge GL (1993) A synaptic model of memory: longterm potentiation in the hippocampus. Nature 361:31-39.
Bliss TVP, Lomo T (1973) Long-lasting potentiation of synaptic transmission in the dentate area of anaesthetized rabbit following stimulation of the perforant path. J Physiol (Lond) 232:331-356.

Böhme GA, Bon C, Stutzmann J-M, Doble A, Blanchard JC (1991) Possible involvement of nitric oxide in long-term potentiation. Eur J Pharmacol 199:379-381.

Böhme GA, Bon C, Lemaire M, Reibaud M, Piot O, Stutzmann J-M, Doble A, Blanchard JC (1993) Altered synaptic plasticity and memory formation in nitric oxide synthase inhibitor-treated rats. Proc Natl Acad Sci USA 90:9191-9194.

Bredt DS, Glatt CE, Hwang PM, Fotuhi M, Dawson TM, Snyder SH (1991) Nitric oxide synthase protein and mRNA are discretely localised in neuronal populations of mammalian CNS together with NADPH diaphorase. Neuron 7:615-624.

Chapman PF, Atkins CM, Allen MT, Haley JE, Steinmetz JE (1992) Inhibition of nitric oxide synthesis impairs two different forms of learning. Neuroreport 3:567-570.

Collingridge GL, Kehl SJ, McLennan H (1983) Excitatory amino acids in synaptic transmission in the Schaffer collateral-commissural pathway of the rat hippocampus. J Physiol (Lond) 334:33-46.

Davis S, Butcher SP, Morris RGM (1992) The NMDA receptor antagonist D-2-amino-5-phosphonopentanoate (D-AP5) impairs spatial learning and LTP in vivo at intracerebral concentrations comparable to those that block LTP in vitro. J Neurosci 12:21-34.

Dwyer MA, Bredt DS, Snyder SH (1991) Nitric oxide synthase: irreversable inhibition by $\mathrm{L}-\mathrm{N}^{G}$-nitroarginine in brain in vitro and in vivo. Biochem Biophys Res Commun 176:1136-1141.

Gallagher M (1985) Re-viewing modulation of learning and memory In: Memory systems of the brain (Weinberger NM, McGaugh JL, Lynch GS, eds), pp 311-334. New York: Guilford.

Garthwaite JS (1991) Glutamate, nitric oxide and cell-cell signalling in the nervous system. Trends Neurosci 14:60-67.

Haley JE, Wilcox GL, Chapman PF (1992) The role of nitric oxide in hippocampal long-term potentiation. Neuron 8:211-216.

Hölscher C, Rose SPR (1992) An inhibitor of nitric oxide synthesis prevents memory formation in the chick. Neurosci Lett 145:165-167.

Huang PL, Dawson TM, Bredt DS, Snyder SH, Fishman MC (1993) Targeted disruption of the neuronal nitric oxide synthase gene. Cell $75: 1273-1286$.

Moncada S (1992) Nitric oxide gas-mediator, modulator, and pathophysiologic entity. J Lab Clin Med 120:187-191.

Morris RGM (1981) Spatial localisation does not depend on the presence of local cues. Learn Motiv 12:239-269.

Morris RGM (1984) Developments of a watermaze procedure for studying spatial learning in the rat. J Neurosci Methods 11:47-60.

Morris RGM, Hagan JJ, Rawlins JNP (1986a) Allocentric spatial learning in hippocampectomised rats: a further test of the "spatial mapping" and "working memory" theories of hippocampal function. Q J Exp Psychol 38B:365-395.

Morris RGM, Anderson E, Lynch GS, Baudry M (1986b) Selective impairment of learning and blockade of long-term potentiation by an $N$-methyl-D-aspartate receptor antagonist, AP5. Nature 319:774-776.

Morris RGM, Davis S, Butcher SP (1990) Hippocampal synaptic plasticity and NMDA receptors: a role in information storage? Philos Trans R Soc Lond [Biol] 329:187-204.

O'Dell TJ, Hawkins RD, Kandel ER, Arancio O (1991) Tests of the roles of two diffusable substances in long-term potentiation - evidence for nitric oxide as a possible early retrograde messenger. Proc Natl Acad Sci USA 88:11285-11289.

O'Keefe J, Nadel L (1978) The hippocampus as a cognitive map. London: Oxford UP.

Schuman EM, Madison DV (1991) A requirement for the intercellular messenger nitric oxide in long-term potentiation. Science $254: 1503$ 1506.

Schuman EM, Madison DV (1993) Nitric oxide and synaptic function. Annu Rev Neurosci, in press.

Shapiro ML, O'Connor C (1992) $N$-methyl-D-aspartate receptor antagonist MK-801 and spatial memory representation: working memory is impaired in an unfamiliar environment but not in a familiar environment. Behav Neurosci 106:604-612.

Vincent SR, Kimura H (1992) Histochemical mapping of nitric oxide synthase in the rat brain. Neuroscience 46:755-784. 